\title{
The Fortress of Riolo Terme, near Ravenna: Digital Survey and 3D Printing for Cultural Dissemination
}

\author{
ELISA SGHERRI, GIOVANNI ANZANI \\ University of Florence, Italy
}

This study is focused on the management of the information derived from the 3D Laser survey of the Fortress of Riolo Terme, near Ravenna, Italy. After studying the building and its history, this survey method was chosen because of its speed in acquiring 3D data, with the advantage of a level of detail and a high definition impossible to obtain by other methods. The geometric survey of the whole fortress and its surroundings was enriched by the collection of color information: taking a specific set of photos at every position, each scan was associated with a panoramic image in order to obtain a point cloud enhanced with the corresponding color values.

Given the complexity and articulation of the building, part of the project was dedicated to the work program, in terms of the decimation of the data, in order to achieve a fluid management of the vast amount of collected data, and the preservation of the appropriate definition of information. This led to the creation of an automatic process both for the alignment of the scans and for the following graphic postprocessing. In addition to the production of drawings as plans, elevations, and sections, whose generation was automated by creation of several procedures for Autodesk AutoCAD, a specific part of the project was aimed at the construction of an optimized model of a portion of the building to be printed out using 3D technology.

The project made it possible to thoroughly investigate an architectural complex that is an outcome of stratifications over time, from the fourteenth century until the present day.

Key words:

Fortress, Digital survey, 3D printing, Masonry, Stratigraphy.

SDH Reference:

Elisa Sgherri, Giovanni Anzani. 2017. The Fortress of Riolo Terme, near Ravenna: Digital Survey and 3D Printing for Cultural Dissemination. SDH, 1, 2, 750-768.

DOI : $10.14434 /$ sdh.v1i2.23228

\section{INTRODUCTION}

The work presented here concerns a digital survey of the Fortress of Riolo Terme, which is situated near Ravenna, Italy. The survey was made with a 3D laser scanner and with use of panorama photography. The panoramic photographs were integrated with the survey results from the scanner to produce point clouds enhanced with color. The first aim of the project was to update the building

Author's address: Elisa Sgherri and Giovanni Anzani, Department of Architecture, University of Florence, Via della Mattonaia, 8 -50121 Florence, Italy; email: sgherrielisa@gmail.com; giovanni.anzani@unifi.it

Permission to make digital or hardcopies of part or all of this work is granted without fee according to the open access policy of SDH.

(C) 2017 SDH Open Access Journal 
information, kept by the municipality, with the addition of accurate and detailed data, creating new documentation of the fortress's condition.

Given the complex articulation of the building and its stratification over time, we considered the traditional forms of representation (such as drawings of plans, sections, and elevations) timeconsuming and often ineffectual in expressing the articulation of the building in its entirety. Therefore, we decided to proceed with both two-dimensional work, by creating a plug-in for AutoCAD to speed up the drawing procedures, and three-dimensional work, by modeling a portion of the building with the goal of producing a physical model in the form of a colored 3D print [Hess and Robson 2010].

In this way, by processing the data collected from the 3D survey (figure 1), we created examples of products that can facilitate understanding of the building and its development over time, while at the same time making it possible to monitor the condition of a historic building. This was possible using only a single instrument, the 3D laser scanner, to which we added the panoramic pictures to increase the type of information we could collect from a survey campaign.

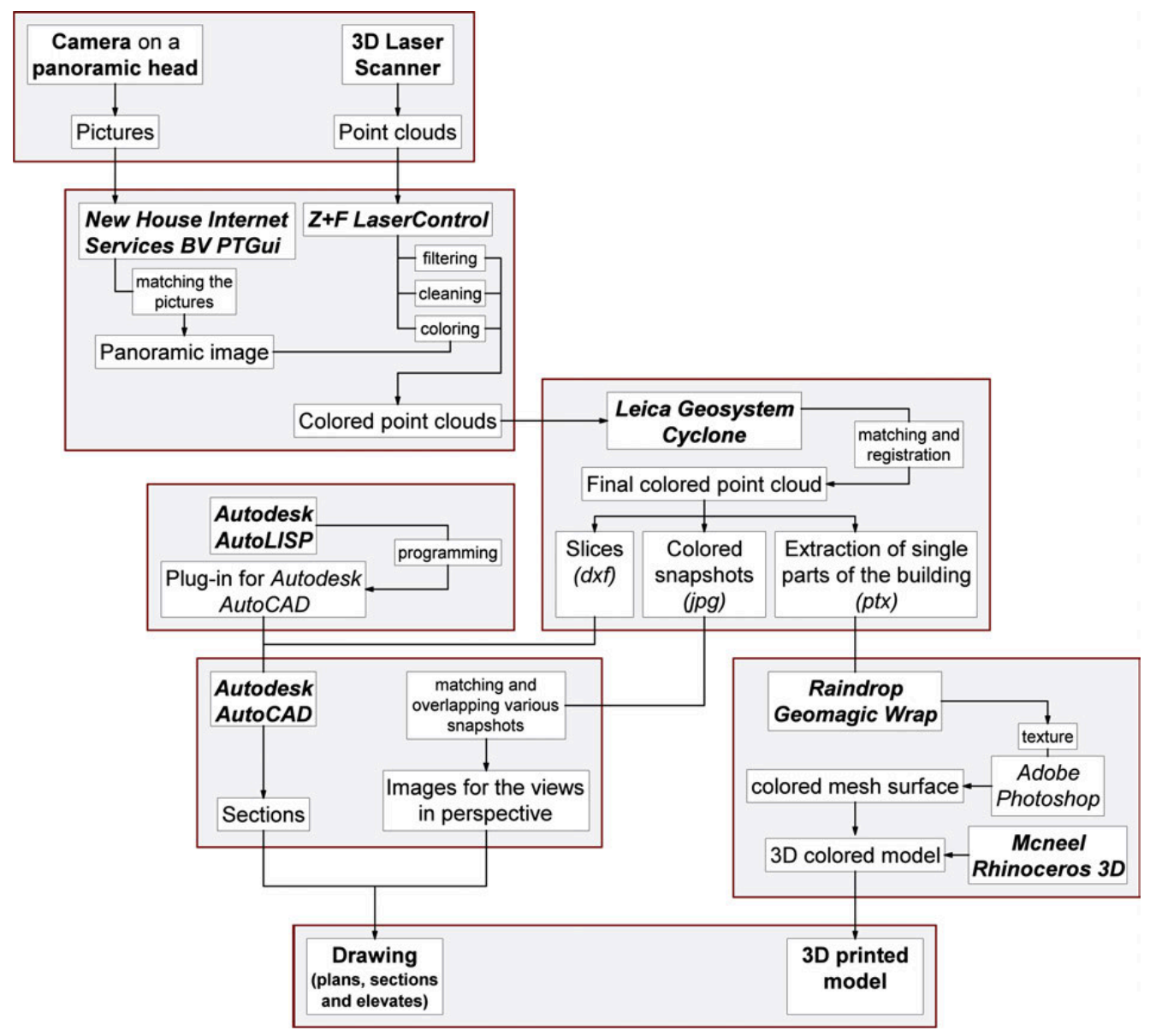

Figure 1. Workflow diagram. Credit: Elisa Sgherri. 


\section{CASE STUDY: GENERAL OVERVIEW}

Riolo Terme is a town of about 6000 inhabitants, located in the hills of the province of Ravenna in central Italy. The first settlement goes back to Roman times, but the town developed mainly in the late fourteenth century, when the territory was contested between nearby Faenza and Bologna. In 1388 the city of Bologna built a fortified tower along with an annexed village [Costa 1992].

The castle underwent various changes and developments until reaching its present shape in the sixteenth century. From this moment, the building lost its military function and remained used for storage until the nineteenth century [Costa 1987]. It is from this period that the fortress began again to be changed: several structures were built leaning against the building, and the square outside was extended by removing the moat.

The period of decline reached its culmination during World War II, since Riolo was located close to a heavily bombed area. Later (1944-1945), the Gothic Line came close to the town, which lay almost along the planned main attack axis of the allies. The great suffering of the whole area left few resources for building maintenance and restoration.

During the last century, the Fortress became property of the municipality and hosted city offices until September 1985. The end of this use signalled the start of interventions aimed at the restoration of the building and the surrounding area [Piersanti and Rava 2000]. The goal was to restore the moat nearly in full and to give the fort the appearance it had had in the sixteenth century (figure 2).

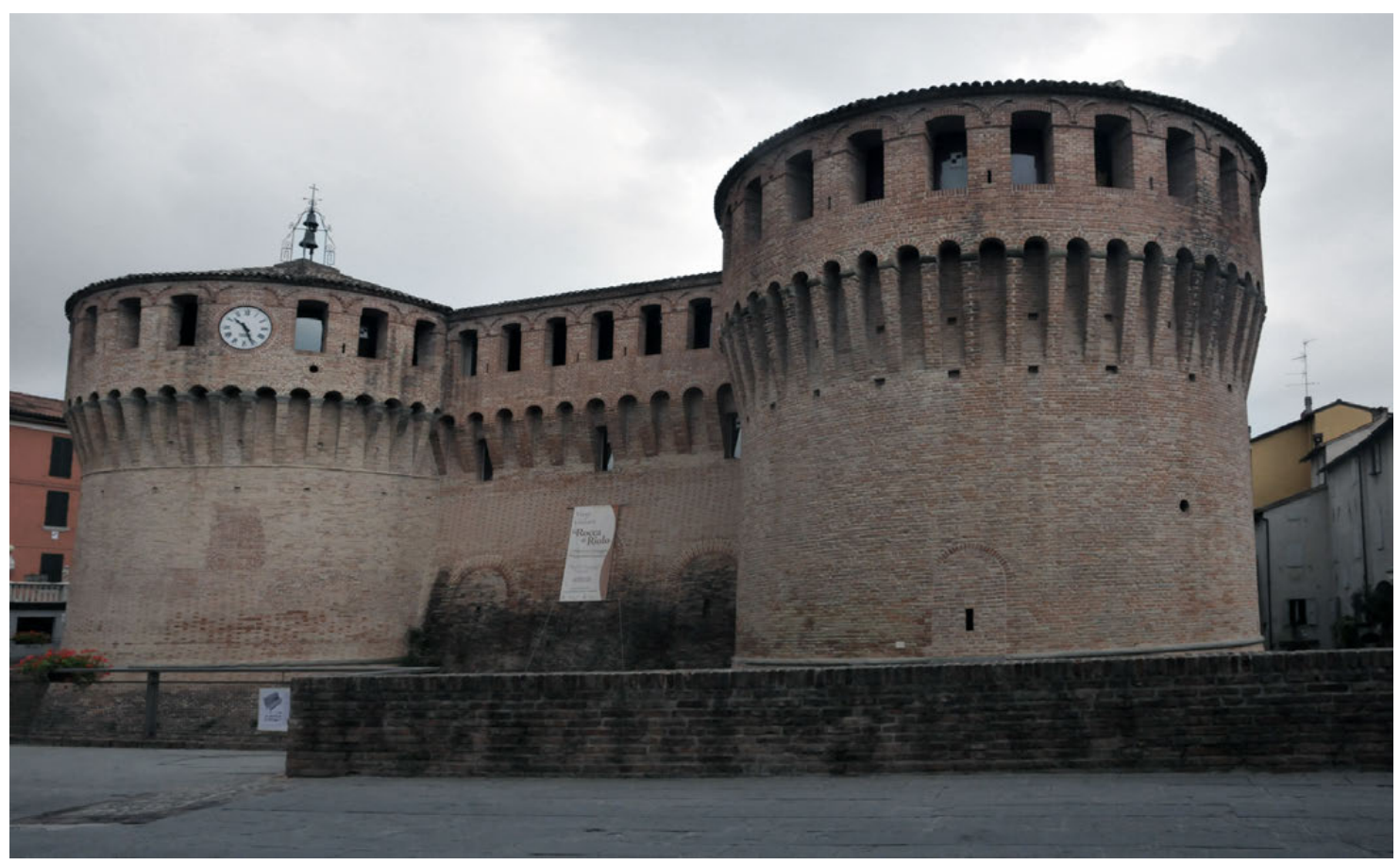

Figure 2. The Fortress of Riolo Terme. Photograph: Elisa Sgherri. 
Currently, the Fortress of Riolo Terme houses several museum exhibits related to the history of the castle [Ancarani et al. 2012]. In addition, many activities are carried out for educational purposes, especially aimed at presenting the history of the place to students from the local schools [Monti 2002].

Such uses of the Fortress motivated the project of restoring and enhancing the quality of the architectural documentation of the building. For this reason, an extended project of research and survey was undertaken to produce new and reliable information. There were two, related goals: to create new drawings useful for the technical operation of the site and also to develop multimedia assets that could integrated into the existing museum exhibits.

\section{ACQUISITION OF THE DATA: THE 3D LASER SCANNER SURVEY}

The survey campaign was conducted over a ten day period by a team of three people. We used the 3D scanner $Z+F$ imager $5006 h$, a device that uses phase-shift technology, with a range of up to $80 \mathrm{~m}$. After acquiring the geometrical data, we carried out the photographic campaign from the same position using a digital camera mounted on a panoramic head. The camera used was a Canon EOS 770D with an 8 Megapixel sensor in CMOS format and mounting a Samyang 8mm Fisheye lens with an angle of $180^{\circ}$ on the diagonal of the frame. The camera and the panoramic head had been conveniently calibrated with the laser scanner so that the two optical centers of the two machines would collimate. In this way, from the same position from which the measurements were gathered, the pictures were also taken. Thanks to the use of a fisheye lens [Wood 1911], with seven pictures (six every $60^{\circ}$ horizontally, and one up) we covered every point detected by the 3D Laser Scanner, with the proper level of overlap needed to assemble the panoramic image [Battini and Bini 2007, Sacerdote and Tucci 2007, Verdiani 2012].

Paper targets were used on the exterior. They were hang over points on the Fortress that we could cover without losing essential data and also on the surrounding buildings so that we obtained a wide base of support for the alignment of each single scan.

As for the interior, it was not always possible to use paper targets because they would cover the wall structure and would not be visible from all the scans that needed to be made, so we used polystyrene spheres with a diameter of $15 \mathrm{~cm}$ [Verdiani 2012], mounted on wooden support, as shown in figure 3. The advantages of this kind of target are numerous: the number of occlusions on the object to be scanned is minimal, and the targets are detectable from a range of $360^{\circ}$, so they are excellent for connecting scans in adjacent rooms [Vassena and Sgrenzaroli 2007].

If on the exteriors the problem was reaching accurate definition of the targets, in the interior we had to pay attention in getting correct overlap of the various scans because the spaces were often narrow and the structure was complex. Moreover, the lack of direct lighting in different rooms created the risks that our photographs would be of poor quality or else even be unusable. 


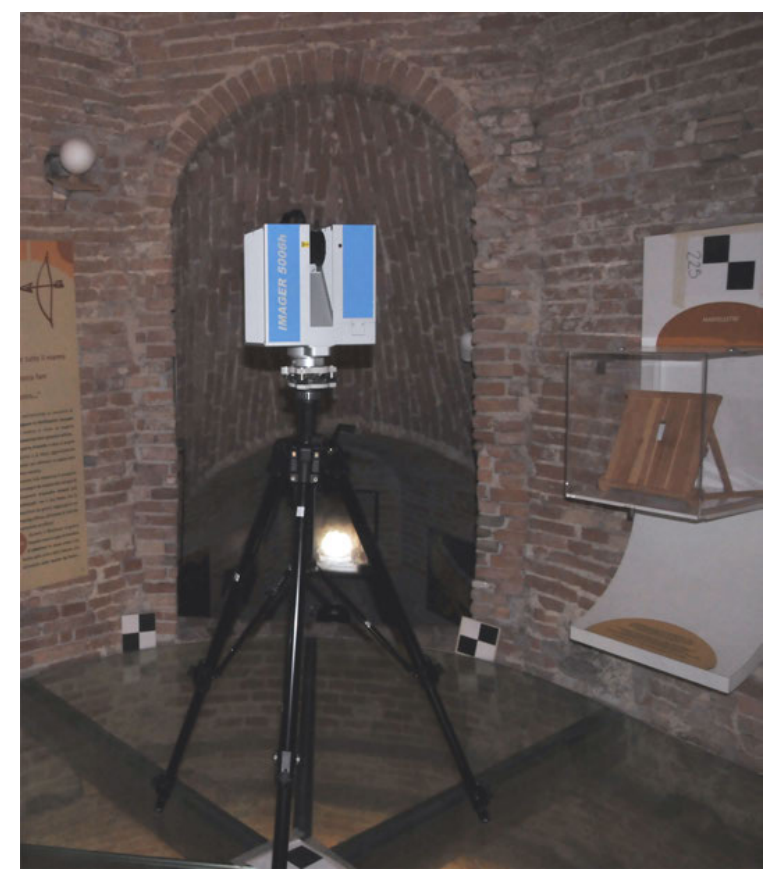

Figure 3. 3D laser scanner with different kinds of targets during the survey campaign inside the Fortress of Riolo Terme. Photograph: Giovanni Anzani.

\subsection{Preparing the workflow}

The greatest difficulty in this project was the data management. The survey produced 296 scans divided between 195 stations, because in numerous cases from the same station we also added details at a higher resolution to obtain good accuracy even for distant features.

Because of the lighting conditions above mentioned, only 148 scans were completed with full panoramic photography. The gaps in our photographic data occurred mostly in secondary rooms, depots and narrow passages. All the main spaces from the interior were geometrically surveyed with high accuracy and mapped with high resolution and quality images.

We later created a database, organized by the nine days of work. Each position was associated with the location on the plan, the information about each scan (such as the settings for resolution accuracy and a note about the contents of the scan), the names of the related images, information about whether the scanning was done with the tilt sensor turned off, and an indication about general position (i.e., if outside or inside).

The latter information helped to calibrate the resolution to use for the scan alignment. In fact, the original data was even too detailed to be consistent with the multimedia purposes and with the scale of many representations, so only in few cases was the original dataset completely used. Thus, the full archive of scans may turn out useful in the future for a specific need, such as checking transformations and deterioration, or simply as a high-quality record of the past condition of the site. To better fit the dataset to our final use, we conducted some tests to decide if we were able to 
streamline the point clouds without losing accuracy, and we decided to halve the number of points for the external scans and to keep every fourth point in the interior. To operate this decimation we applied a filter during the export from the $Z+F$ Laser Control software. In this way, we obtained a lighter dataset, a prerequisite for a more fluid management of all the collected data, with no compromise of accuracy and with a variable level of detail according to the importance and/or complexity of the single part.

\subsection{Processing point clouds}

Once the management of the collected data was terminated, we proceeded to process the point clouds.

The purpose of the survey campaign was to obtain scans of the Fortress and its surroundings, which, in addition to the information on the reflectance of the material, as usual, also contained information on the color of the buildings. This was possible by producing, for each position, a set of photos merged into a single panoramic image and then associated with the scan.

Seven pictures, captured from the same position, were joined to create a single panoramic image thanks to semi-automatic processes of the New House Internet Services BV PTGui software, as shown in figure 4 . This software manages to create a wider picture from single ones, if these have a good amount of points in common. After the matching is done, both with automatic and manual procedures, the edges of the pictures are blended to obtain a homogeneous result.

The image thus obtained was exported in jpg format with a ratio of height-width of 1: 2 that was equivalent to that of the scans [Sfikas et al. 2016].

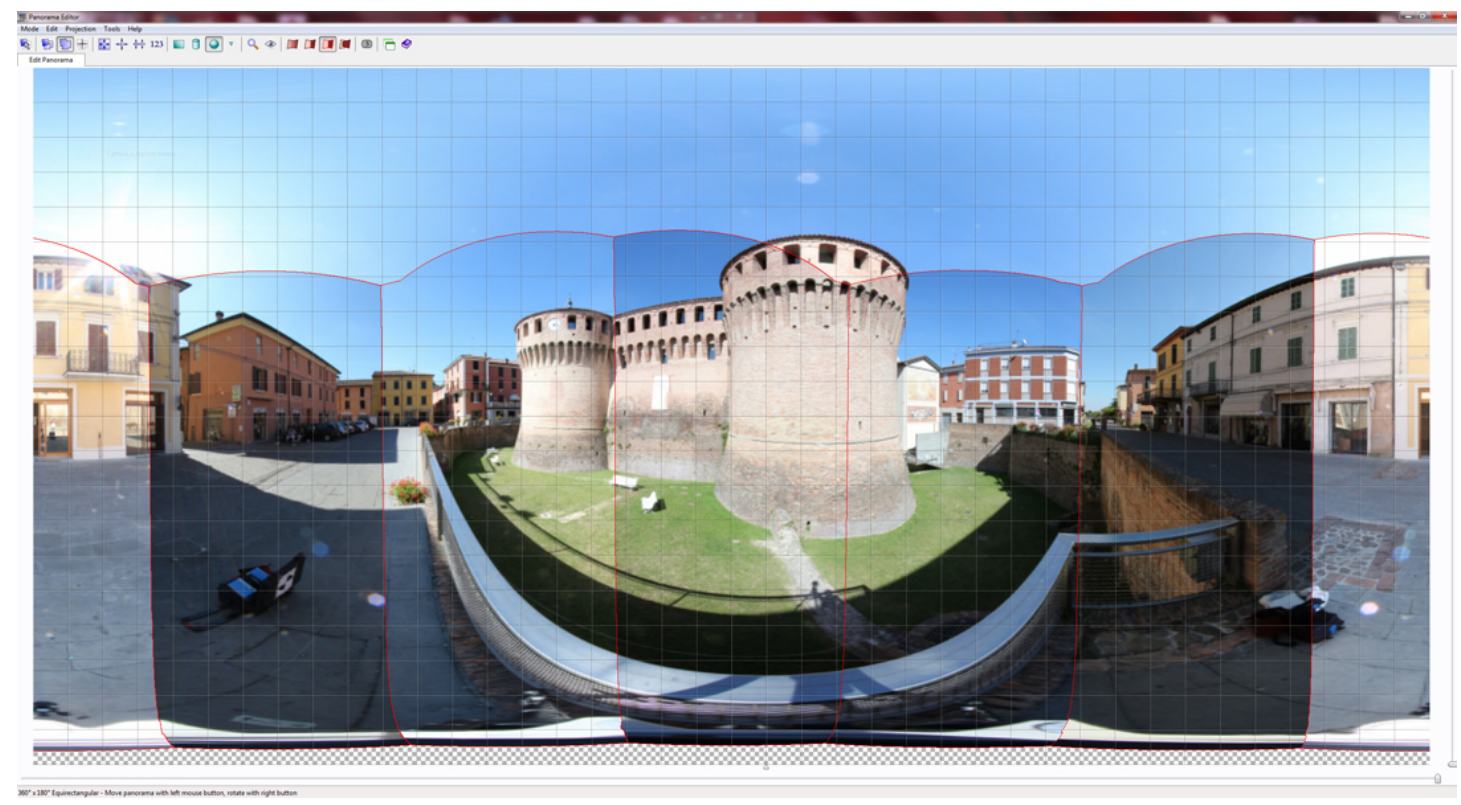

Figure 4. Panoramic image created from seven single pictures. Credit: Elisa Sgherri. 
The problem of working with a panoramic head is that, taking pictures at $360^{\circ}$ from the same point, it may happen that some of those will be overexposed or underexposed. To reduce these effects and have a more homogeneous image, individual photos were processed using Adobe Photoshop, where some pictures were made brighter or darker to better match with their group. After this procedure, the pictures were reloaded into the New House Internet Services BV PTGui software, and this result in a better overlap.

Once all the pictures had been prepared, we proceeded to match them with the point clouds using the $Z+F$ Laser Control software (figure 5), thanks to a command that made the process automatic. In some cases, however, this was not sufficient, and we had to proceed manually with the matching of at least four corresponding points for each pair of image and scan.

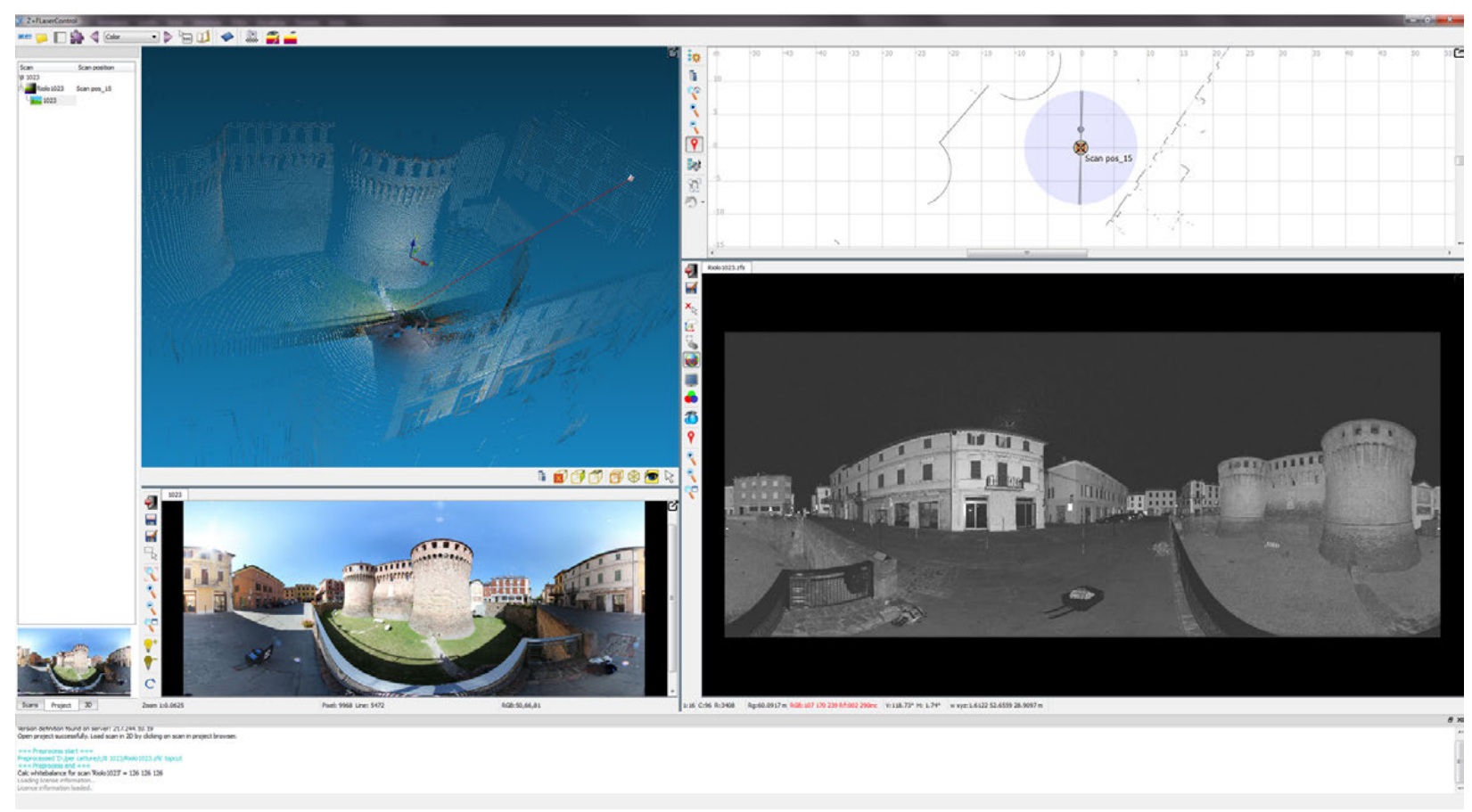

Figure 5. Coloring a scan through the Z+F Laser Control software. Credit: Elisa Sgherri.

Another task carried out by the $Z+F$ Laser Control software was cleaning the point clouds (figure 6). In the process of scanning we had worked near reflective surfaces that caused the creation of some artefacts inside the point clouds, such as small groups of points with no reference to real object and characterized by quite dark grayscales. This effect appears because the laser reacts to the surface in relation to the reflection of the material, so with object like metal, mirrors or glasses the measurement can be not accurate and some erroneous data may appear in the resulting point cloud. These erroneous areas, clearly identifiable in a single scan, could overlap corrected points during the registration phase. 


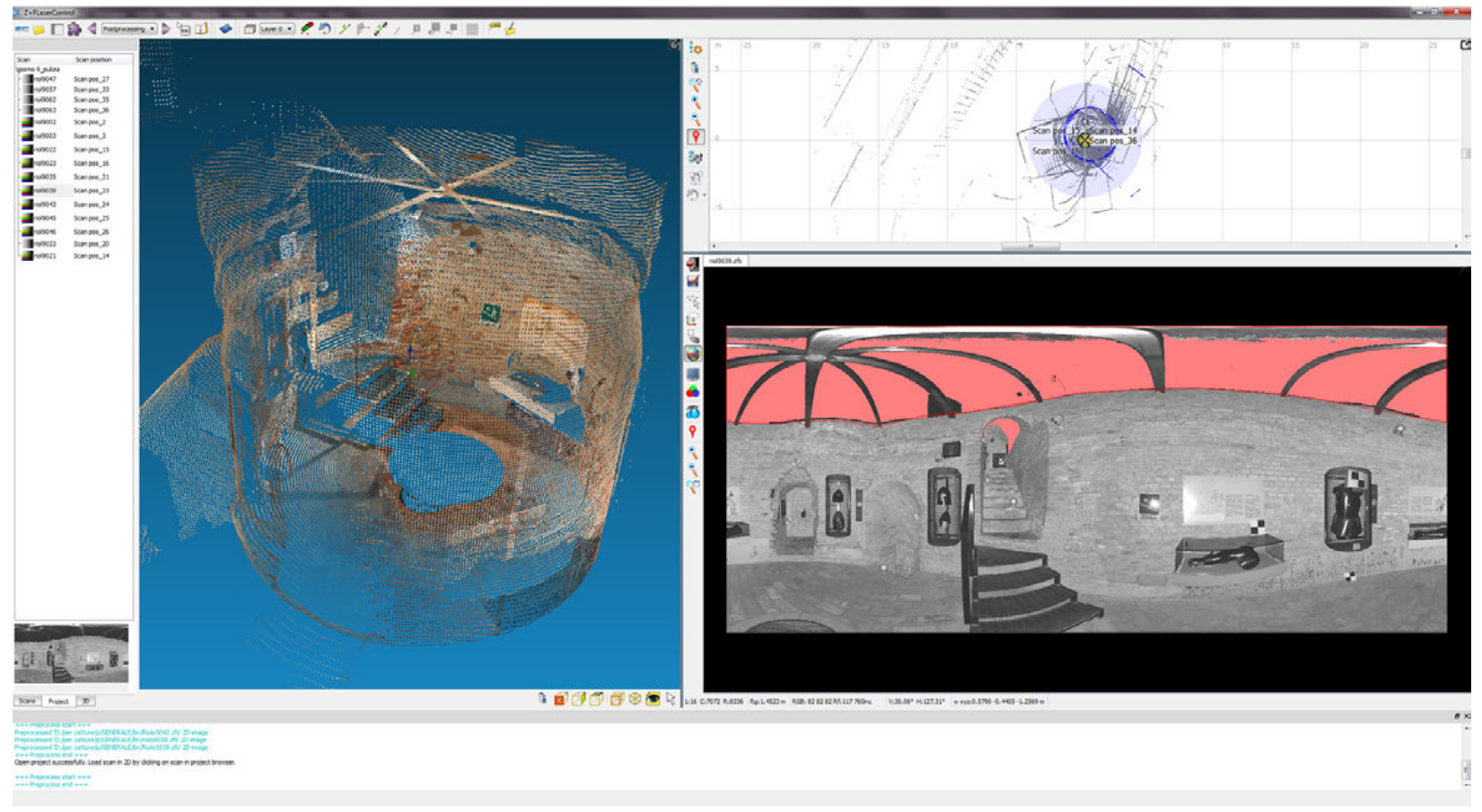

Figure 6. Cleaning a scan with the Z+F Laser Control software. Credit: Elisa Sgherri.

After all these processes, the point clouds were exported for later processing with Leica Geosystem Cyclone software. This software was used to check every scan, naming and registering the targets visible in the resulting point cloud, and then matching two or more scans using common targets. Each scan had its own reference system, corresponding to the center of the scanner, and after the registration of the matching, the software created a single point cloud that carried the reference system of the scan chosen as the leading one.

The matching and registration of individual scans was carried out with the help of several databases organized according to the days and type of scan. In this way, the size of the individual working files was reduced, allowing a more fluid management of the data.

At this point, our survey project was divided into nine IMP files, containing the total scans, which would be mainly used for further work, and nine of detail, to provide targets for more accurate registrations.

Since for every day we had up to forty-five scans, and each of them contained various targets (figure 7), it would have been impossible to decide which ones were the best pairs for recordings only by comparing the data collected. 


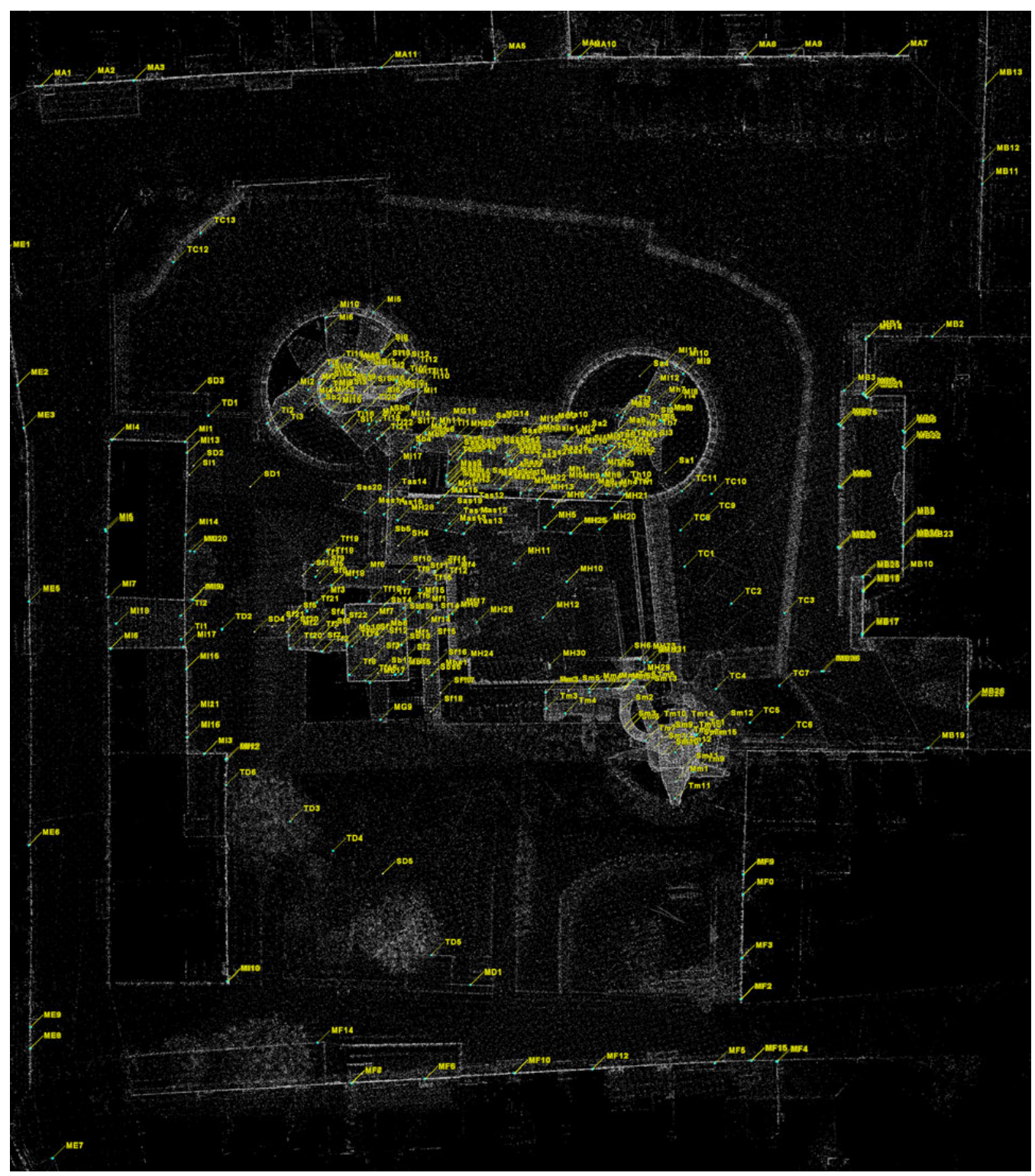

Figure 7. View, from the top, of the final point cloud with the display of the targets used for the registrations. Credit: Elisa Sgherri.

By using Microsoft Office Excel, we created a chart for every IMP file, which made it possible easily to cross-check the information about scans and targets. Then, by using some internal macro, we counted the number of common targets, and we used this information to create a new comparison chart. Thanks to this display, it was easier to identify which matching was the best one, choosing scan pairs or triads with the highest number of common targets. At this point, we chose the pairs or triads of scans to register. After deciding which one was the best match, we checked if the registration phase in Leica Geosystem Cyclone would give the same result. 
Regarding the recordings on the interior, we never went over $0.005 \mathrm{~m}$. for errors on constraints, disabling those targets that exceeded the maximum threshold. This procedure was not always automatic, especially about the external scans: having to choose which constraints to ignore, we preferred to maintain reliable the targets applied on the Fortress, and disable those on the surrounding buildings. Thus, in the case of unavoidable errors, we decided to have better overlap of the scans on the Fortress to the detriment of precision on the surrounding buildings.

Before confirming each registration, we also applied the Cloud Constraint, which is the comparison of two scans through surface matching, and the addition of this comparison as a constraint. Since this is the most reliable type of constraint, we controlled that the errors on it never exceeded 0.002 $\mathrm{m}$.

Since we had already exported some test plans to verify the accuracy of the survey, it was clear how difficult the choice of the height would be to obtain plans or sections that were interesting and readable. Therefore, we decided first to analyze the plans. We sectioned the building every $0.5 \mathrm{~m}$ and chose among the plans those that best would describe each of the building's five floors. Each plan was then composed of a mosaic of several horizontal sections, since every area or room had different changes in altitude.

In addition, thanks to the tests already conducted, we knew that the optimum thickness of the slices to be exported in DXF was $0.001 \mathrm{~m}$. Those slices were later made two-dimensional in Autodesk AutoCAD. While exporting, we left active the original reference system so that each of the plans was referenced to the others.

Once we obtained the plans, we chose two main directions, orthogonal to each other, for the vertical sections, and we then proceeded to export about one hundred slices. Since the work on point clouds had generated a colored model (figure 8), we chose to use this data to produce images to be associated with the elevation views.

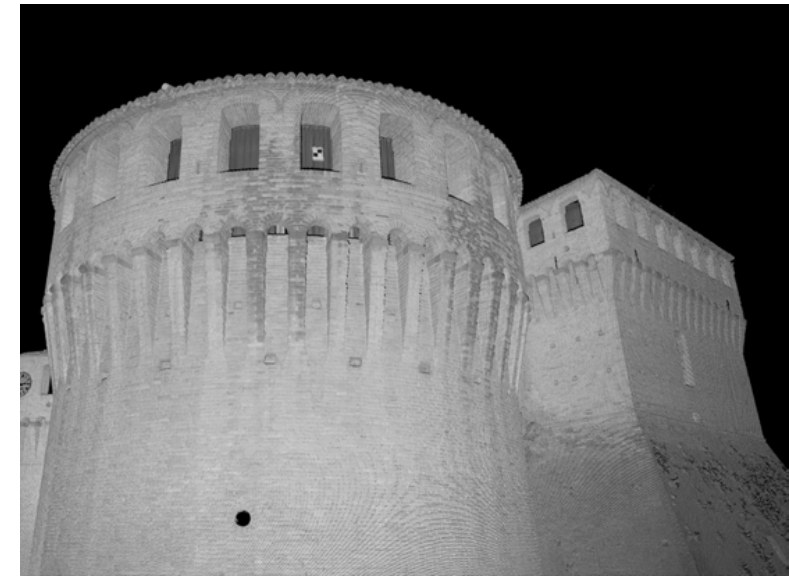

a

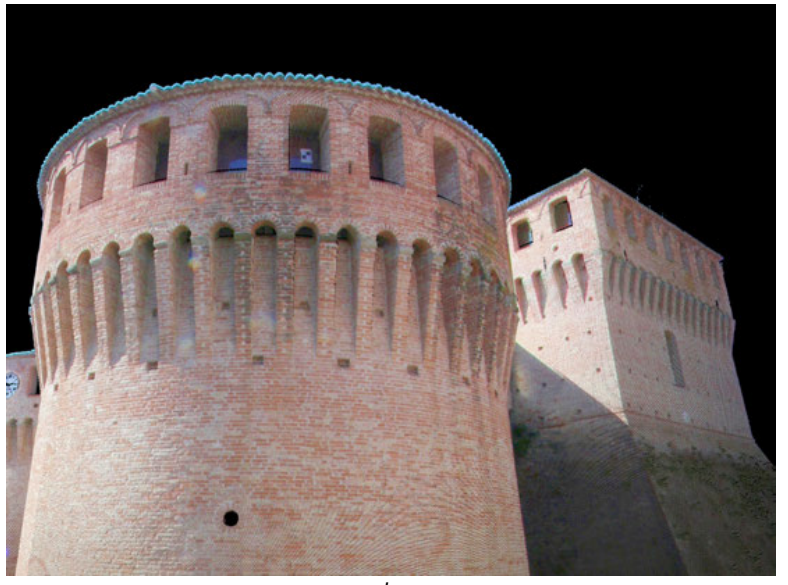

b

Figure 8. Difference between the traditional point cloud display (a) and the colored one (b) in Leica Geosystem Cyclone software. Cred: Elisa Sgherri. 
This was possible by creating several snapshots in Leica Geosystem Cyclone, which are captures from the screen view of a portion of the selected point cloud.

Once we obtained all the necessary snapshots, these were assembled together through New House Internet Services BV PTGui software. In Adobe Photoshop, in contrast, we curated the editing phase of the images by correcting inevitable errors arising from the resolution of the data collected. We decided to not hide the lack of homogeneity of some snapshots owing to the different density of point clouds, or to their lack of color. The work thus obtained was superimposed onto the sections processed in Autodesk AutoCAD, integrating this drawing with all the missing details not visible in a section.

\section{RESULTS}

Our principal goal was the creation of products that would help us to better understand the complexity of evolution of the building as a series of interventions over the centuries. We wanted to create different kinds of output starting from the same data. To accomplish this, we decided that it was necessary to speed up some of the post-production processes of point clouds to guarantee a fluid workflow, but still to maintain the accuracy appropriate for a technical work as well as the comprehensibility of a product intended for a larger audience. We decided to produce both two- and three-dimensional products, working on the point clouds, taking advantage of the chromatic information added to the geometrical one [Pavlidis et al. 2007].

For the two-dimensional work, we decided to create several plans, elevations, and sections to show the complexity of the structure in its entirety, adding images from the colored point clouds to show the original materials and the masonry texture (figure 9).
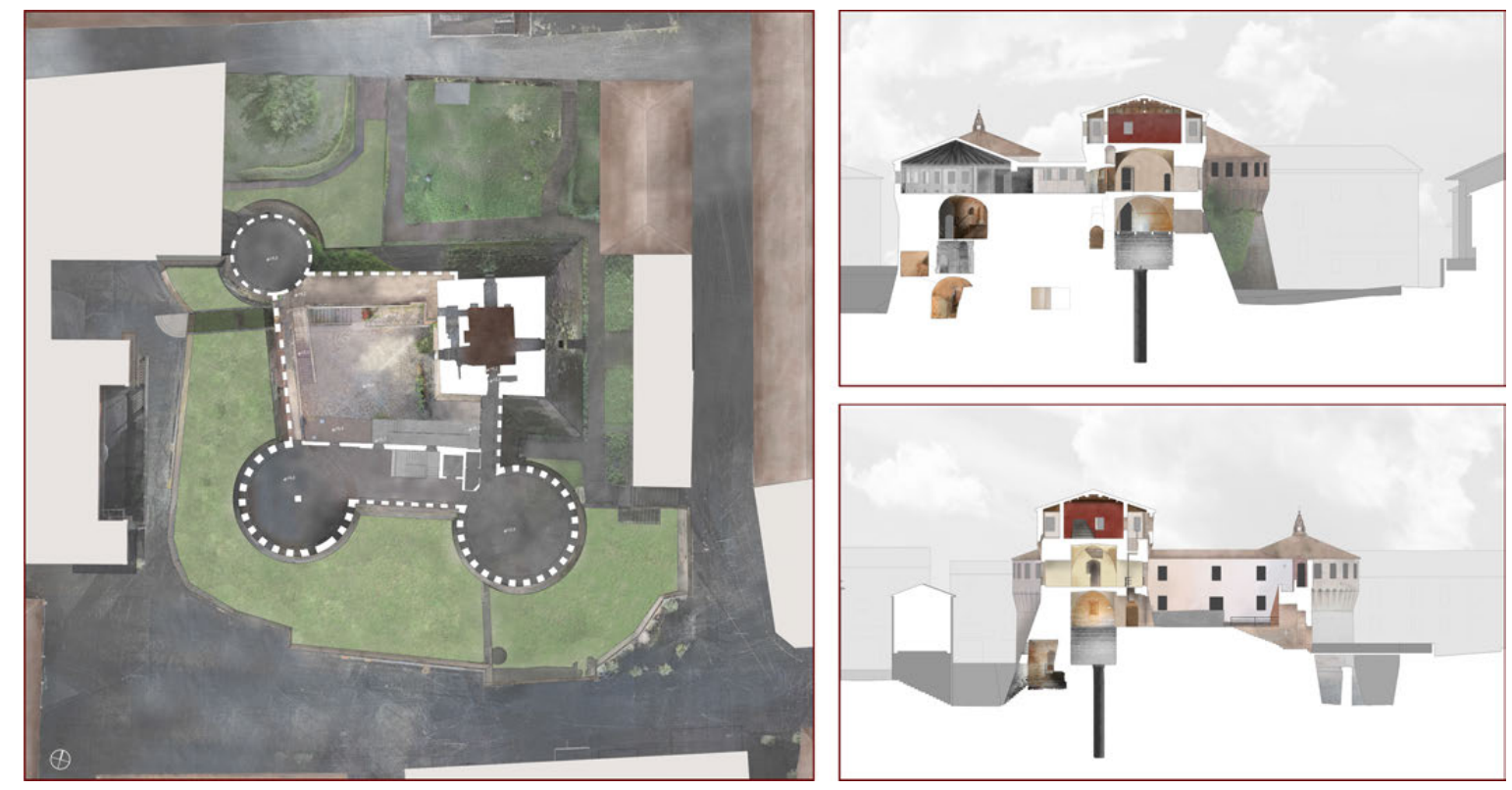

Figure 9. Examples of final plans and sections. Credit: by Elisa Sgherri. 
For the three-dimensional product, it was decided to create a physical model using 3D printing binder technology. We then decided to study a cross-section of the main tower to show both the interior and the exterior as well as the relationship between the bulk of the structure and the narrowness of the spaces.

\subsection{Two-dimensional work: set up of the AutoLISP plug-in}

After we had obtained plans and sections that were still slices of point clouds exported from Leica Geosystem Cyclone, the next step was to process this data to make it editable in Autodesk AutoCAD. Part of the research was the compilation of a plug-in for this software to make this procedure as fast and accurate as possible. We considered hand drawing in a such an imposing work to be inappropriate. We developed a series of applications in AutoLISP format to obtain plans and sections.

The first step was to convert the information from points to polylines. Therefore, we created a command that achieves this goal, which could detect the change of direction or curvature of the final polyline. This has been possible thanks to the hand drawing of a very coarse polyline, called "trajectory." Selecting the points, already made two-dimensional, that describe the edge of the section, and indicating the trajectory polyline, the command ideally projected the various points onto the nearest segment of the trajectory, and used this as a reference to orient the polyline portion that had to be drawn. The result was a polyline that passed through all the selected vertices in the order provided by the trajectory. The command could be used in any type of User Coordinate System (UCS) because the information is automatically translated into global coordinates. If points and trajectory are not coplanar, the command will draw a polyline that is located on a plane parallel to the xy current UCS, passing from the first point selected, and which have as trajectory the projection of this on the plane of the polyline (figure 10).
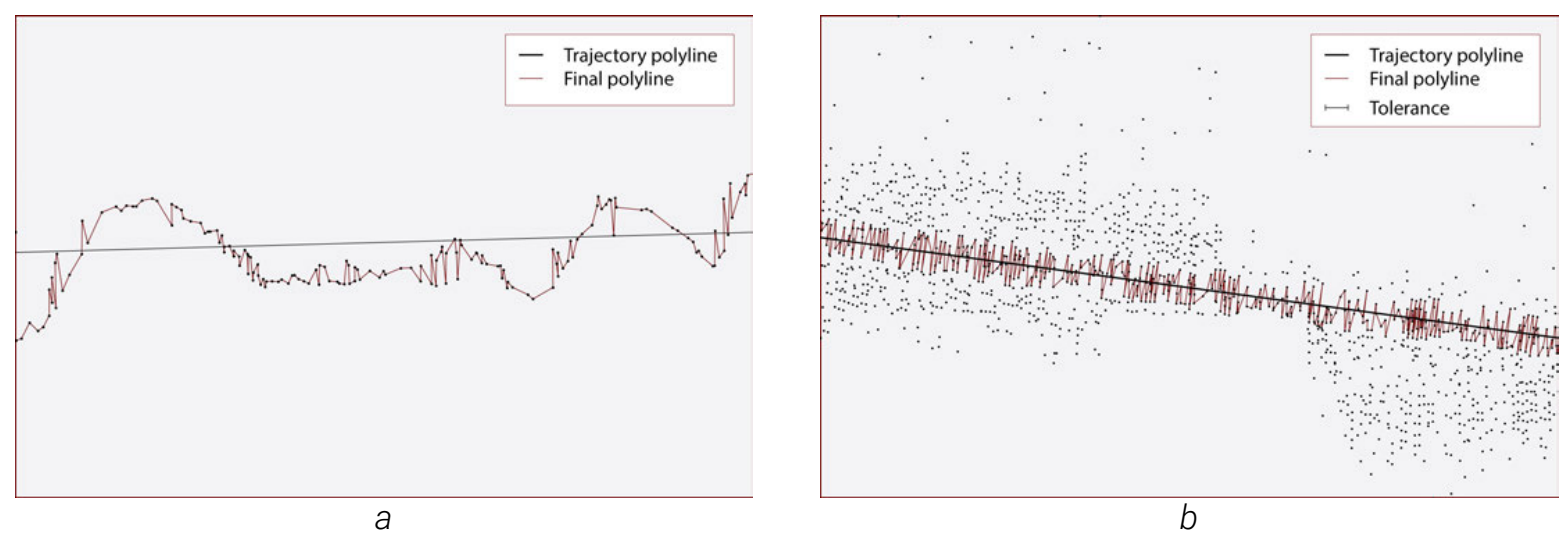

Figure 10. Portions of two different polylines passing by the vertices of a point cloud. One polyline (a) is created only by following the lead of the trajectory, for the drawing of the other (b) also a tolerance range has been used. Credit: by Elisa Sgherri.

The function can be used recursively if the polyline obtained is not sufficiently precise. In fact,it is possible to add vertices or to move existing ones in the trajectory and then to re-run the command. This automatically recognizes an active selection set and asks the operator if he wants to use the 
previous data. This is quite useful in the case of very large sets of selections, because to get a more accurate result is sufficient to just edit a few details of the trajectory polyline. The function returns a result very quickly, since it does not recalculate the data already computed in the previous step.

An implementation of this function is a command that works with a tolerance range for the distance of the points from polyline trajectory. In fact, if the survey is not sufficiently precise, and therefore the slice is thick, or in the case where the section plane is oblique to some object, resulting in a spot of points and not an edge, it can be useful to isolate a range of points to be included in the calculation of the new polyline (figure 10).

Instead of selecting only the desired vertices, it is enough to draw the trajectory appropriately and to define a maximum distance for the points to be calculated. It is also very helpful to exclude random points, which are not always visible and not necessary for the drawing and often inappropriate in the definition of the section. This command works on each type of reference system, but points and trajectory must be coplanar since the distance between them is calculated over the three dimensions.

Once the drawing of the section is obtained, this is very heavy because the polyline will have as many vertices as the number of the selected points. It is therefore necessary to decimate the data without altering the readability of the section. We decided to use the Douglas-Peucker procedure, already known and used in various fields as an algorithm for simplification of sets of lines, since it returns the best representation of an original curve in terms of perception. This algorithm is based on the discretization of a polyline, starting from the maximum simplification, which is the segment that combines the initial vertex to the end of the polyline, as shown in figure 11 ( $1^{\text {st }}$ step). The next step is to add the farthest point from the created segment, and that will be inserted between the vertices of the starting polyline, creating the new simplified polyline. At each iteration of the procedure, therefore, the distance of the input vertex is calculated from the partial optimization polyline, and the point at the greater distance is added. The process ends when the distance to the farthest point is less than a tolerance value decided in advance [Douglas and Peucker 1973].
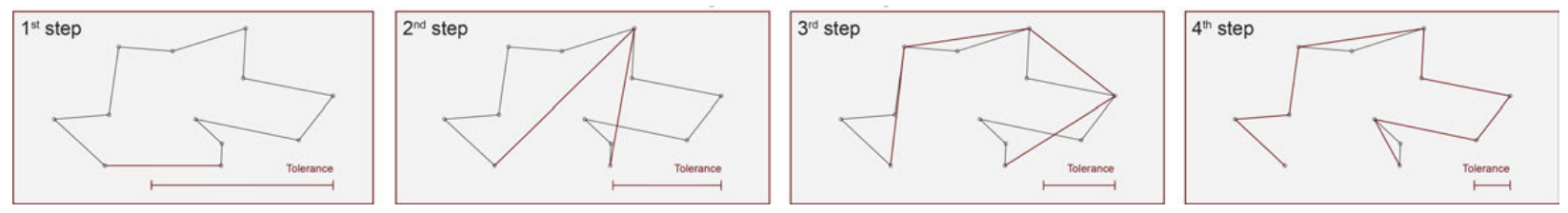

Figure 11. Example of the development, in four steps, of the Douglas-Peucker procedure. Credit: Elisa Sgherri.

One of the main problems of this procedure is to avoid self-intersection of the lightweight polyline that is more likely if the tolerance values are high [Wu and Rocìo Gonzales Màrquez 2003]. Thanks to the tools of the AutoLISP language, we overcame this problem because every input point is recorded and sorted, obtaining a polyline where the vertices maintain the correct order. Before the beginning of the process, the operator will be asked to select the polyline to discretize and the tolerance value. From here, the recursive procedure that uses the Douglas-Peucker algorithm begins. If the tolerance value entered is too high to get a good approximation, it is possible to repeat the procedure, using another command that employs the calculation already executed, computing only the points 
excluded because they were too far, thus speeding up the processing times [Hershberger and Snoeyink 1992]. Both commands work exclusively within the global coordinate system. The result is a polyline that describes the object as well as the original one, as shown in figure 12 , but, since it contains a very reduced number of vertices, permitting a much more fluid work.
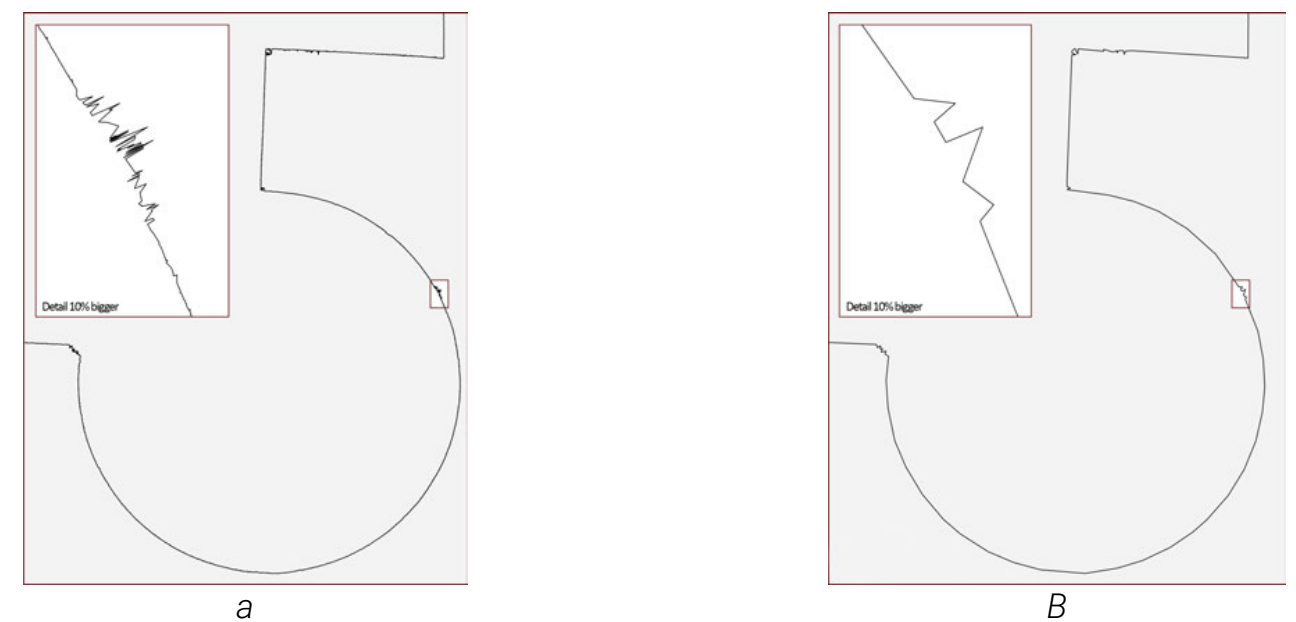

Figure 12. Portion of the same plan created with different tolerances on the procedure. One polyline (a) is made from 14874 vertices, the other (b) has 142 vertices. Credit: Elisa Sgherri.

\subsection{Three-dimensional work: modeling for 3D printing}

In addition to the creation of the drawings, we also made a physical model in order to facilitate the public's understanding of the building.

Since the data acquired during the survey campaign also contained the color information, we designed a model made using 3D printing binder technology with gypsum powder as a base material. As with 3D printers that utilize other technologies, the execution is carried out through deposition of layers. In this case the base material, after being deposited, is made integral with a glue. If the model has also color information, the machine will utilize a combination of glues, tinged with pigments, to obtain the corresponding color details. The resolution obtained by the survey was very high so we decided to focus only on a portion of the building, working at the scale of 1:100, as was the case with the drawings.

For the sample chosen, since the size of the print area was limited, we decided to print out a crosssection of the main tower, called "mastio", which is the oldest part of the Fortress. Starting again from the data created using Leica Geosystem Cyclone software, we chose the portion of the building to be printed, then the object was split between external and internal scans for an easier follow-up process [Pieraccini et al. 2001]. The scans which had no color information were kept for those spaces where it was not possible to capture images, while they were ignored where the colored point clouds were sufficient to describe the object.

The selected data were then exported as PTX format to keep the color information, and they were processed through the Raindrop Geomagic Wrap software [Böhm and Pateraki 2006]. When 
importing, for both interior and exterior, all the data were kept, and we chose to apply a filter only later. In this software the scans are recognized individually, so the first step was to join all the data.

We applied the first filtering in order to eliminate isolated points or others that were not sufficiently close to the main object, because they could create noise in the following phases. The points were then registered to the same grid so the data were as homogeneous as possible. The consecutive step was to convert the information, up to now expressed in points, in to mesh (figure 13). This was done semi-automatically with the Wrap command: the software analyzes the points and translates them into triangles. To the single mesh is connected chromatic information, which is the result of the average RGB values belonging to its vertices [Barrile and al. 2017].
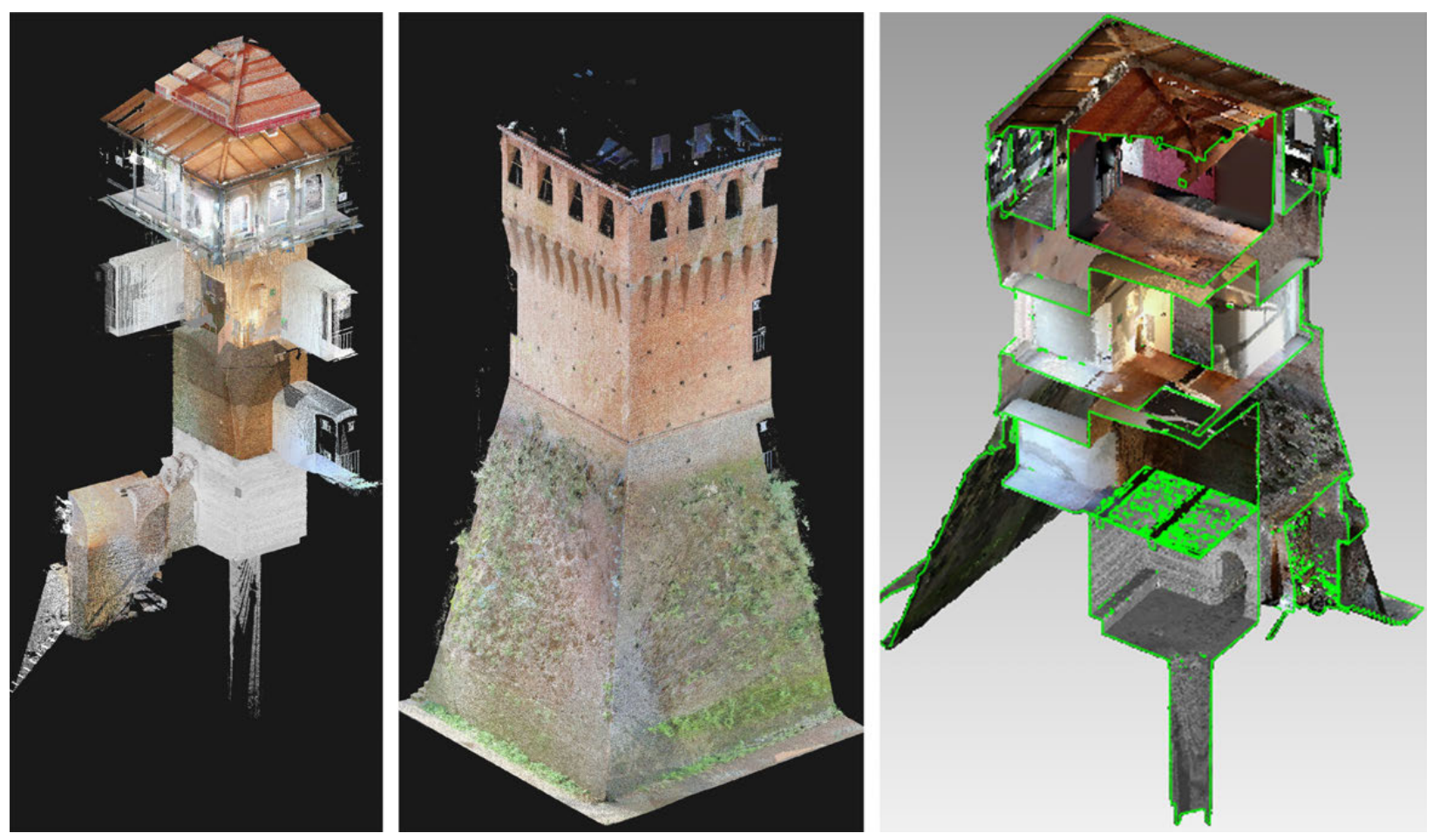

Figure 13. Transforming data from point clouds to mesh with Raindrop Geomagic Wrap software. Credit: Elisa Sgherri.

Before proceeding with further processing and simplification, we generated the texture associated with the model, which is the development, as a plane image, of the color data of the entire object. Once we obtained the set of surfaces that described the model, for the interior of the building a further subdivision into portions was required. We decided to aim for smaller regions both to reduce the data and to be able to better control the model in each area.

The creation of the mesh was a semi-automatic process so the obtained surfaces did not describe the object in its entirety. Because of the building irregularities and the non-homogeneity of the original point clouds, the surface was not continuous. 
Through both automated and manual processes, all the gaps were then filled (figure 14), by eliminating holes or double surfaces.
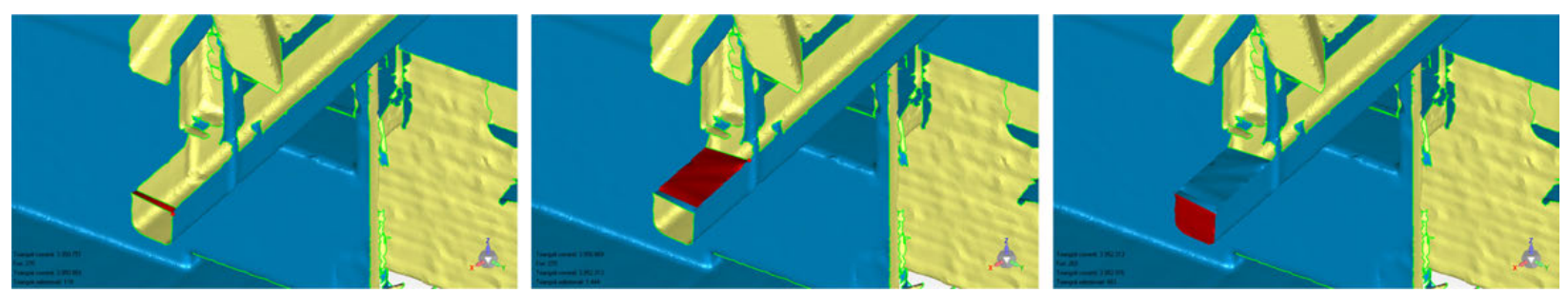

Figure 14. Examples of procedures to repair the surface manually. Credit; Elisa Sgherri.

A further problem encountered was the lack of information in some portions of the model. By studying the building, we could replicate the missing data in different ways: where the object was regular (for example, there were some gaps in a few openings that were identical to another) the surface was integrated into the Raindrop Geomagic Wrap software, meanwhile in other areas it was necessary to use additional software.

There were a few regions, such as the roof, where the point clouds were so rarefied that it was not possible to obtain a correct mesh, even though it had been sufficient to produce two-dimensional drawings. We used this information to obtain the missing portions, for example by modeling the roof tiles through the software Mcneel Rhinoceros 3D. Once this procedure was done, the new portion was imported in Raindrop Geomagic Wrap and matched with the rest of the surface. Since the simplification of the model in these regions has been perceptible, we chose to highlight the separation from the main body by printing these portions without the relative color.

The last task in Raindrop Geomagic Wrap was the union of the various parts into a single object. At this point, the data were too heavy to be controlled in an easy way, so we decided to eliminate all the information that was unnecessary because of the scale of the final print. We proceeded then to the decimation of the mesh from 12 million to 5 million polygons.

Since the texture presented some imperfections that would not facilitate the understanding of the object, we decided to correct the image associated with the model in a separate process, using Adobe Photoshop. In this way, the gap, owing to the association of photos taken with different light, was reduced, and we tried to limit the loss of color information arising from the operations on the model.

Once printed, the model was cleaned from the supporting base material and treated with Cyanoacrylate bond (figure 15) to obtain brighter colors and a more resistant object. 


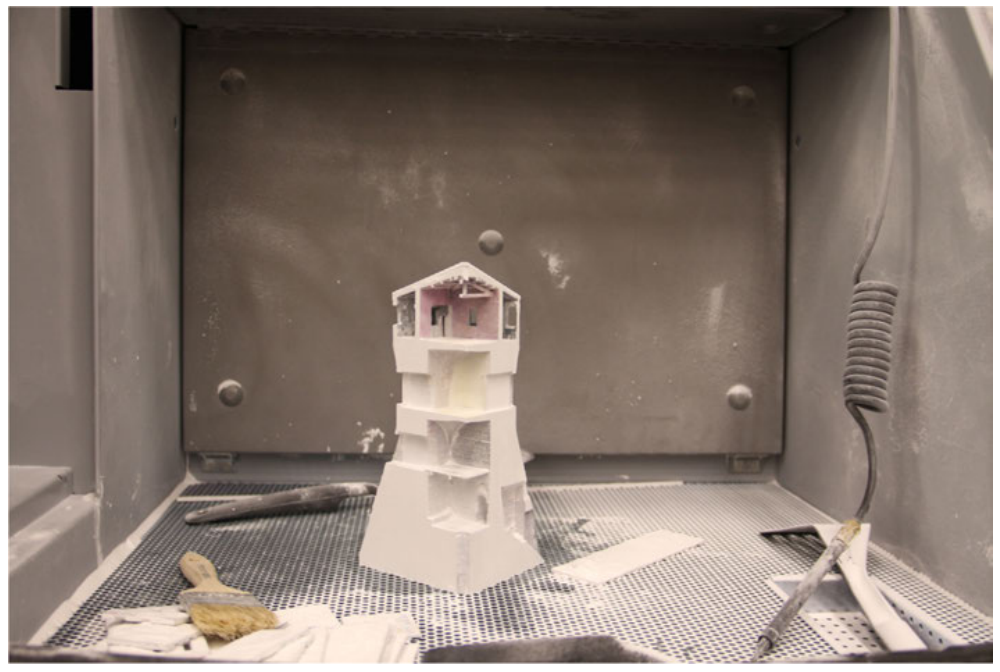

a

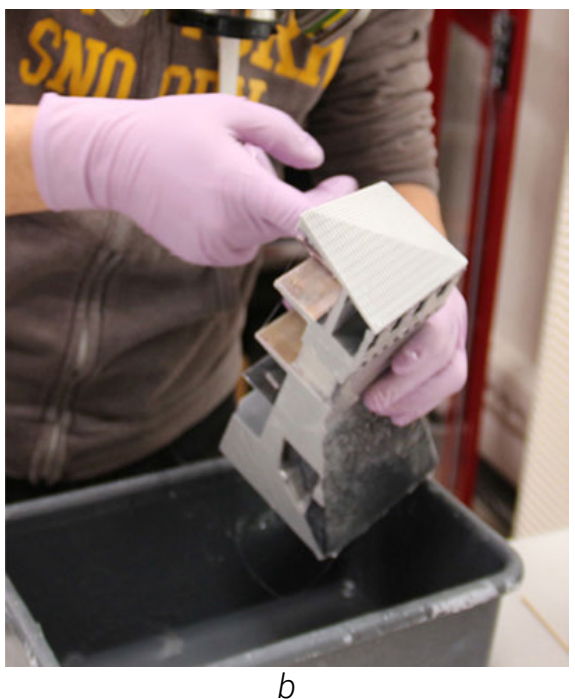

Figure 15. The model in the 3D printer (a), for cleaning off the extra powder, and (b) during the treatment with Cyanoacrylate bond. Credit: Elisa Sgherri.

The high resolution of the survey led us to create a very accurate and detailed model. The masonry texture is perceptible even at the scale 1:100, also thanks to the printing technology chosen. The model accurately represents the articulation of the building and gives an example of the bulk of the structure, typical of fortresses.

\section{CONCLUSIONS}

The starting point of this work was a data collection, through a survey campaign, which allowed us to monitor the condition of an historical building with the goal of both thorough study and creation of products that would help us to better understand, and later show to the general public, the development of the structure over time.

We needed to create a series of products that were at once accurate enough for technical use but still comprehensible for wider public dissemination.

The use of colored point clouds is what allowed us to develop these results. In fact, by adding the chromatic data to the scans, we built a database from which it was possible to obtain images or threedimensional models that were more useful than the traditional ones.

The accuracy we can obtain with modern machines allowed us to capture every detail of this building complex, and we were able to create a reliable database of the condition of the building. We also wanted to use the same data collected in a single survey to create tangible outputs for this work. The aim was to have a single database that was suitable for different kinds of further processing and to create a workflow that allowed us to easily use a single resource to obtain several products suited for various purposes. Therefore, as the survey techniques turned from manual to semiautomatic, we 
wanted to do the same for the further processes, both for the matching of the point clouds and the process of drawing.

The possibility of adapting the level of detail, and consequently the processing time, to the kind of output or resolution needed often speeded up the process, because only the data that truly affect the needed display was processed.

This was our aim in adopting the Autodesk AutoCAD plug-in, and also our goal in processing the digital model that led to the 3D print.

The facility of collecting data typical of modern instruments still requires a human operator able to choose the appropriate resolution of the work and to balance the accuracy and fluidity of the workflow.

\section{REFERENCES}

Ancarani, L., M. Morigi and S. Tamburini. 2012. Museo del paesaggio dell'appennino faentino, Ravenna: Angelo Longo Editore.

Barrile, V., G. Bilotta, D. Lamari. 2017. 3D models of Cultural Heritage, International Journal of Mathematical Models and Methods in Applied Sciences 11, 2017, 1-8.

Battini C. and M. Bini. 2007. Nuove immagini di monumenti fiorentini. Rilievi con tecnologia scanner laser 3D, Firenze: Alinea.

Böhm J. and M. Pateraki. 2006. From Point Samples to Surfaces - On Meshing and Alternatives, ISPRS Commission V Symposium 'Image Engineering and Vision Metrology', 2006, 50-55.

Costa, L. 1987. Aspetti inediti di storia romagnola, Il castello di Riolo e gli ultimi pertinaci paladini di Caterina Sforza, Faenza: Tipografia faentina.

Costa, L. 1992. Nascita e sviluppo di un castello medioevale, Torricelliana, 266-297.

Douglas, D. and T. Peucker. 1973. Algorithms for the Reduction of the Number of Points Required to Represent a Digitized Line or its Caricature, The Canadian Cartographer 10(2) (1973), 112122. DOI: 10.3138/FM57-6770-U75U-7727

Hershberger, J. and J. Snoeyink. 1992. Speeding-up the Douglas-Peucker Line-Simplification Algorithm, University of British Columbia Vancouver, BC, Canada.

Hess, M. and S. Robson. 2010. 3D colour imaging for cultural heritage artefacts, International Archives of the Photogrammetry, Remote Sensing and Spatial Information Sciences - ISPRS Archives 38 (PART 5), 288-292.

Monti, A. 2002. Riolo e la sua Rocca, Appunti di storia e d'archeologia, Imola: Bacchilega Editore.

Pavlidis, G., A. Koutsoudis, F. Arnaoutoglou, V. Tsioukas, C. Chamzas. 2007. Methods for 3D Digitization of Cultural Heritage, Journal of Cultural Heritage 8 (2007), 93-98.

Pieraccini, M., G. Guidi, C. Atzeni. 2001. 3D Digitizing of Cultural Heritage, Journal of Cultural Heritage 2 (2001), 63-70.

Piersanti, C. and R. Rava. 2000. La rocca ritrovata, il restauro del complesso fortificato di Riolo Terme, Milano: Skira.

Sacerdote, F. and G. Tucci. 2007. Sistemi a scansione per l'architettura e il territorio, Firenze: Alinea. Sfikas, K., I. Pratikakis, A. Koutsoudis., M. Savelonas, T. Theoharis. 2016, Partial Matching of 3D Cultural Heritage Objects Using Panoramic Views, Multimedia Tools and Applications, April 
2016. DOI: $10.1007 / \mathrm{s} 11042-014-2069-0$

Vassena, G. P. M. and M. Sgrenzaroli. 2007. Tecniche di rilevamento tridimensionale tramite laser scanner, Milano: Selecta.

Verdiani, G. 2012. Rilievo digitale 3D, le metodologie e le strumentazioni per il rilievo laser scanner, Tipologie delle strumentazioni per il rilievo digitale. In: S. Bertocci, M. Bini. Manuale di rilievo architettonico e urbano, Novara: Città studi Edizioni, De Agostini, 169-197.

Wood, R. W. 1911. Physical Optics, The Macmillan Company, New York.

Wu S.-T. and M. Rocìo Gonzales Màrquez. 2003. A Non-self-intersection Douglas-Peucker Algorithm, SIBGRAPI2003, 60-66.

Received March 2017; revised July 2017; accepted August 2017. 